This item was submitted to Loughborough's Research Repository by the author.

Items in Figshare are protected by copyright, with all rights reserved, unless otherwise indicated.

\title{
Three dimensional modelling of interaction between surface and Darcy flow regimes through soils
}

\section{PLEASE CITE THE PUBLISHED VERSION}

http://dx.doi.org/10.2166/wst.2009.577

\section{PUBLISHER}

(C) IWA Publishing

\section{VERSION}

NA (Not Applicable or Unknown)

\section{LICENCE}

CC BY-NC-ND 4.0

\section{REPOSITORY RECORD}

Kaveh-Baghbaderani, Bahareh, Vahid Nassehi, and A. Kulkarni. 2009. "Three Dimensional Modelling of Interaction Between Surface and Darcy Flow Regimes Through Soils”. figshare. https://hdl.handle.net/2134/5424. 
This item was submitted to Loughborough's Institutional Repository (https://dspace.lboro.ac.uk/) by the author and is made available under the following Creative Commons Licence conditions.

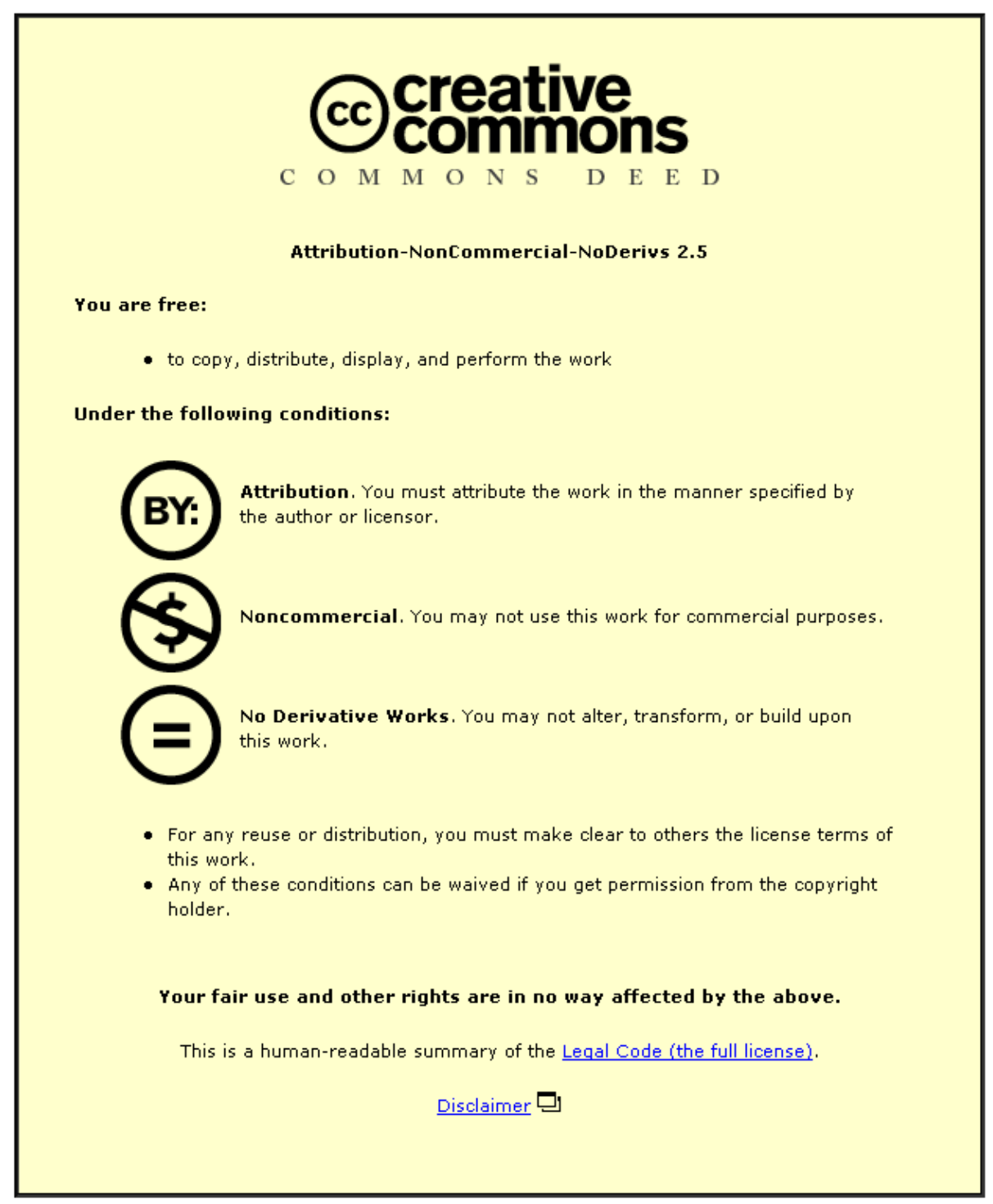

For the full text of this licence, please go to: http://creativecommons.org/licenses/by-nc-nd/2.5/ 


\title{
Three dimensional modelling of interaction between surface and Darcy flow regimes through soils
}

\author{
B. Kaveh-Baghbaderani, V. Nassehi, A. Kulkarni
}

Chemical Engineering Department, Loughborough University, Loughborough LE11 3TU, UK

(Email:B.Kaveh-Baghbaderani2@lboro.ac.uk)

\begin{abstract}
The present paper deals with the impact of surface flow on hydrodynamic conditions in saturated underground domains. A three dimensional finite element scheme has been used to simulate underground flow resulting from the flow of water over a saturated land. The results clearly show the effects of the surface flow on the hydrodynamic conditions of the subsurface porous regions. This analysis is an important prerequisite for the prediction of contaminant mobility in soils and hence provides a convenient tool for the prediction of interaction between surface and subsurface flow processes. For low permeability cases, considered here, the governing equations consist of water continuity and Darcy equations. These equations are solved using a robust and reliable finite element procedure.
\end{abstract}

KEYWORDS Underground flow; Hydrodynamics; Darcy flow; Finite element modelling; Three dimensional porous flow.

\section{INTRODUCTION}

Seepage in soils is an essential topic of study in many civil engineering and environmental protection processes. For example, in the design of earth dams and retaining structures, that require quantification of drainage, amount of seepage need to be identified. Similarly seepage is the determining factor in the contaminants mobility as leachates in subsurface domains. Seepage flow models have been developed by many researchers (e.g. see Reddi, 2003; Das and Nassehi, 2002; Cedergren, 1994). These investigations have shown that seepage regimes often have complicated characteristics mainly because of the heterogeneity of soils through which water flows. Heterogeneity of soil media arises for various reasons such as the presence of staggered layers of soil with different porosity. Therefore reliable mathematical description and modelling of seepage flow requires formulation of realistic features of the problem and the boundary conditions affecting the flow. Transport equations describing flow through porous media depend on the properties of the fluid and factors such as permeability and porosity and overall structure of media (Das and Nassehi, 2001; Bear, et al. 1991). In this regard it is important to distinguish between saturated and unsaturated domains. Seepage flow in unsaturated lands has been the subject of many studies (e.g. Troldborg, 2009; Uromeihy, 2007). However, unlike the unsaturated situations, the influence of surface flow on saturated domains has not been widely studied. This may be due to the common belief that surface flow makes only small contribution to the changes of hydrodynamic conditions under the ground in saturated domains. The aim of this paper is to show that there is a significant link between surface flow and subsurface conditions in saturated lands. Simulations obtained in this work can therefore be considered as quantitative analysis of the link between surface and subsurface flows for saturated cases. Rain water flowing over the surface of saturated lands is the main cause of interaction between surface flow and a porous regime under the ground.

The main factors affecting flow through porous soils are hydraulic gradient and the coefficient of soil permeability. Permeability is a function of the range of grain size and shape, stratification, consolidation and cementation of the material. The rate of flow is commonly assumed to be directly proportional to the hydraulic gradient, however this is not always true under realistic conditions. 
Theoretically, due to the increasing load, permeability of soils decreases with increasing depths. Therefore a layered heterogeneous strata is the common feature of flow domain in most environmental studies. In table 1 a typical range of soil permeability used in the present study is shown. These data are based on previously published experimental and field survey results (Bell, 2000 and Lage, 1998).

\begin{tabular}{lcl}
\hline Degree of permeability & $\begin{array}{c}\text { Range of coefficient of } \\
\text { Permeability }\left(\mathbf{m}^{2}\right)\end{array}$ & \multicolumn{1}{c}{ Soil type } \\
\hline High & $10^{-6}$ & $\begin{array}{l}\text { Medium and coarse gravel } \\
\text { Medium }\end{array}$ \\
$10^{-6}$ to $10^{-8}$ & $\begin{array}{l}\text { Fine gravel; coarse; medium and fine sand; } \\
\text { dune sand; clean sand - gravel mixtures }\end{array}$ \\
low & $10^{-8}$ to $10^{-10}$ & $\begin{array}{l}\text { Very fine sand, silty sand, loose silt, loess, } \\
\text { well fissured clays }\end{array}$ \\
Very low & $10^{-10}$ to $10^{-12}$ & $\begin{array}{l}\text { Dense silt, dense loess clayed silt, poorly } \\
\text { fissured clays } \\
\text { Un- fissured clays }\end{array}$ \\
Impermeable & $10^{-12}$ & in
\end{tabular}

Table 1- Range of soil permeability

Establishment of porous flow under the ground is the result of fluid system pressure imbalance in lands. The underground fluid system pressure is often expressed as the hydraulic head. In order to obtain quantitative predictions for underground flow it is therefore necessary to represent these regimes via equations involving the hydraulic head. Quantitative relationships between velocity, pressure and elevation heads can be obtained using mass, momentum and force balances. However, velocity head in soils is usually negligible and in majority of cases the main driving force can be assumed to result from the combination of elevation and pressure heads. This term can be simply represented by a single pressure term. Therefore saturated flow in a porous medium can be modelled using either the Darcy (Darcy, 1856); or the Brinkman (Brinkman, 1947) equations depending on the permeability/porosity of the medium and flow Reynolds number (Wakeman and Tarleton, 2005). For very low Reynolds number (creeping flow) and porosity less than 0.6 , the most suitable equation is the Darcy equation. This is the dominant situation in most types of seepage flow of water in soils. In a previous paper we demonstrated the validation of the Darcy's law for isotropic, homogenous, incompressible, saturated and isothermal porous media (Kulkarni, et al. 2008). The Darcy equation inherently implies perfect slip conditions at domain boundary walls and does not include any wall effects (Ishizawa and Hori, 1966). Therefore an accurate solution scheme for this equation should be capable of yielding a slip velocity on the walls of porous domains. The finite element scheme used in the present work can very effectively cope with such boundary conditions. This technique can also take into account irregular geometries. However, considering the large scale of lands where environmental phenomena needs to be studied, any irregularity of the domain walls can be ignored. In this work we have used a block as a representative section of a typical saturated land where an underground flow regime is established because of rain water flowing over its surface.

\section{MODEL EQUATIONS}

The governing model equations of the seepage flow of an incompressible Newtonian fluid such as water are represented as:

\section{Equation of continuity}

The continuity equation (i.e. expression of conservation of mass) for an incompressible fluid is represented (using vector notations) as: 


$$
\nabla \cdot \vec{u}=0
$$

Where $\vec{u}$ is the velocity vector.

\section{Equation of flow}

As mentioned earlier in this work we have selected the Darcy equation to represent the flow equation (i.e. expression of conservation of momentum). Using vector notation this equation (Nield and Bejan, 1992) is written as:

$$
\rho \frac{\partial \vec{u}}{\partial t}+\nabla p+\frac{\mu}{\vec{K}} \cdot \vec{u}=0
$$

Where $\rho$ is fluid density, $p$ is the pressure, $\mu$ is viscosity of the fluid and $\overrightarrow{\vec{K}}$ is the permeability of the porous medium. In its most general description $\overrightarrow{\vec{K}}$ should be regarded as a second order tensor which is represented, in a matrix form, as:

$$
\vec{K}=\left[\begin{array}{ccc}
K_{x x} & 0 & 0 \\
0 & K_{y y} & 0 \\
0 & 0 & K_{z z}
\end{array}\right]
$$

Where $K_{x x}, K_{y y}$ and $K_{z z}$ are the principle components of the permeability tensor along the $x, y$ and $z$ directions of a Cartesian coordinate system. Any anisotropy in a porous medium can hence be taken into account by assigning appropriate values to the components of the permeability tensor. Conjunctive solution of equations (1) and (2) poses a mathematical problem as the first equation does not include a pressure term. Full mathematical analysis of the problem is somewhat obscure and requires lengthy explanations. However, it has been shown that a stable and accurate solution for these equations can be obtained provided that the solution scheme satisfies a condition known as the LBB condition (Reddy, 1986). A convenient way of satisfying this condition is to replace equation (1), which is the expression of incompressibility, with a modified form (Zienkiewicz and Wu 1991) as:

$$
\frac{1}{\rho c^{2}} \frac{\partial P}{\partial t}+\nabla \cdot \vec{u}=0
$$

Where $c$ is the speed of sound in the fluid. Equation (4) represents conservation of mass for a slightly compressible fluid.

\section{BOUNDARY CONDITIONS}

In the present work simulations of underground flow of water are obtained by the conjunctive finite element solution of equations (2) and (4) in a three-dimensional domain subject to the following boundary conditions:

1. Top surface: Constant velocity tangent to the surface.

2. Side walls: Perfect slip conditions.

3. Bottom surface: Perfect slip conditions.

4. Downstream surface: Zero pressure set as an arbitrary datum.

On the slip surfaces the normal component of the velocity is set to be zero whilst the tangential components are calculated as unknowns by the model. 


\section{MATHEMATICAL MODEL}

The governing equations of the present model are solved using a finite element scheme based on the mixed UVWP technique(Zienkiewics, 2005). The problem domain is divided (discretized) into a computational grid consisting of finite elements. The finite elements used in this discretization are 8 node three dimensional hexahedrons. To develop the working equations of the present scheme the field variables are approximated using the shape (interpolation) functions associated with three dimensional 8 node hexahedral elements as follows:

$$
\begin{array}{ll}
u \approx \tilde{u}=\sum_{j=1}^{k} N_{j} u_{j} & v \approx \tilde{v}=\sum_{j=1}^{k} N_{j} v_{j} \\
w \approx \tilde{w}=\sum_{j=1}^{k} N_{j} w_{j} & p \approx \tilde{p}=\sum_{j=1}^{k} N_{j} p_{j}
\end{array}
$$

Where $N_{j}$ is the shape function associated with node $\mathrm{j}(j=1, \ldots ., 8)$ and $u_{j}$ etc. are the nodal values of field variables (i.e. degrees of freedom). As already mentioned the use of equation (4) allows the substitution of both pressure and velocity by approximations formed by the same order shape functions in the present scheme. After this substitution the obtained residuals are weighted using shape functions in the usual manner prescribed by the standard Galerkin finite element method (Zienkiewics, 2005). This leads to the derivation of elemental working equation as:

$$
\left[\begin{array}{cccc}
A_{j}^{11} & A_{j j}^{12} & A_{j j}^{13} & A_{j j}^{14} \\
A_{j}^{21} & A_{j}^{22} & A_{j j}^{23} & A_{j j}^{24} \\
A_{j}^{31} & A_{j j}^{32} & A_{j j}^{33} & A_{j j}^{34} \\
A_{j}^{41} & A_{j j}^{42} & A_{j j}^{43} & A_{j j}^{44}
\end{array}\right]^{24}\left\{\begin{array}{c}
U_{j} \\
V_{j} \\
W_{j} \\
P_{j}
\end{array}\right\}^{n+1}=\left\{\begin{array}{c}
C_{j} \\
C_{j} \\
C_{j} \\
C_{j}
\end{array}\right\}^{n}
$$

Where

$A_{i j}^{11}=\iiint_{\Omega_{e}}\left(N_{i} N_{j}+\alpha \Delta t \frac{\mu}{\mathrm{K}_{x} \rho} N_{i} N_{j}\right) d x d y d z$

$A_{i j}^{12}=0$

$A_{i j}^{13}=0$

$A_{i j}{ }^{14}=-\iiint_{\Omega_{e}} \alpha \Delta t N_{j} \frac{\partial N_{i}}{\partial x} d x d y d z$

$A_{i j}{ }^{21}=0$

$A_{i j}^{22}=\iiint_{\Omega_{e}}\left(N_{i} N_{j}+\alpha \Delta t \frac{\mu}{\mathrm{K}_{y} \rho} N_{i} N_{j}\right) d x d y d z$

$A_{i j}^{23}=0$

$A_{i j}^{24}=-\iiint_{\Omega_{e}} \alpha \Delta t N_{j} \frac{\partial N_{i}}{\partial y} d x d y d z$

$A_{i j}^{31}=0$

$A_{i j}^{32}=0$

$A_{i j}^{33}=\iiint_{\Omega_{e}}\left(N_{i} N_{j}+\alpha \Delta t \frac{\mu}{\mathrm{K}_{z} \rho} N_{i} N_{j}\right) d x d y d z$

$A_{i j}^{34}=-\iiint_{\Omega_{e}} \alpha \Delta t N_{j} \frac{\partial N_{i}}{\partial z} d x d y d z$

$$
\begin{aligned}
& A_{i j}^{41}=\iiint_{\Omega_{e}} \alpha \Delta t c^{2} N_{i} \frac{\partial N_{j}}{\partial x} d x d y d z \\
& A_{i j}^{42}=\iiint_{\Omega_{e}} \alpha \Delta t c^{2} N_{i} \frac{\partial N_{j}}{\partial y} d x d y d z \\
& A_{i j}^{43}=\iiint_{\Omega_{e}} \alpha \Delta t c^{2} N_{i} \frac{\partial N_{j}}{\partial z} d x d y d z \\
& A_{i j}^{44}=\iiint_{\Omega_{e}} N_{i} N_{j} d x d y d z \\
& C_{j}^{1}=-\int_{A_{e}} \alpha \Delta t N_{j} P_{j}^{e} n_{x} d A_{e} \\
& C_{j}^{2}=-\int_{A_{e}} \alpha \Delta t N_{j} P_{j}^{e} n_{y} d A_{e} \\
& C_{j}^{3}=-\int_{A_{e}} \alpha \Delta t N_{j} P_{j}^{e} n_{z} d A_{e} \\
& C_{j}^{4}=-\iint_{A_{e}}\left(\frac{1}{2} \alpha \Delta t^{2} c^{2}\right) N_{j} \frac{\partial P_{j}^{e}}{\partial x} n_{x} d A_{e} \\
& -\int_{A_{e}}\left(\frac{1}{2} \alpha \Delta t^{2} c^{2}\right) N_{j} \frac{\partial P_{j}^{e}}{\partial y} n_{y} d A_{e} \\
& -\int_{A_{e}}\left(\frac{1}{2} \alpha \Delta t^{2} c^{2}\right) N_{j} \frac{\partial P_{j}^{e}}{\partial z} n_{z} d A_{e}
\end{aligned}
$$


Note that superscripts $(n+1)$ and $n$ in equation (6) represent the new and old time steps. Members of the elemental equation (6) are transformed into a local elemental coordinate system using isoparametric mapping. This allows the utilization of a uniform numerical integration process such as Gauss-Legendre quadrature to obtain values of definite integrals appearing in the member terms of the working equations corresponding to all of the elements in the computational grid (Reddy and Gartling, 1994). After the evaluation of the terms of the elemental working equations, they are assembled over the common nodes in the computational grid to obtain the system of algebraic global stiffness equations. After the prescription of the boundary conditions, this system becomes determinate and can be solved using a frontal solution method (Irons 1970). The described simulations are carried out using an in-house developed Intel Visual FORTRAN code in Pentium IV processor. This has been implemented in previous paper(Kulkarni, et al, 2008) The results are plotted using Techplot 360 ® (evaluation version).

\section{SOLUTION ALGORITHM}

Steps required to complete a solution cycle via the described scheme can be summarised by the following flow diagram (Figure 1).

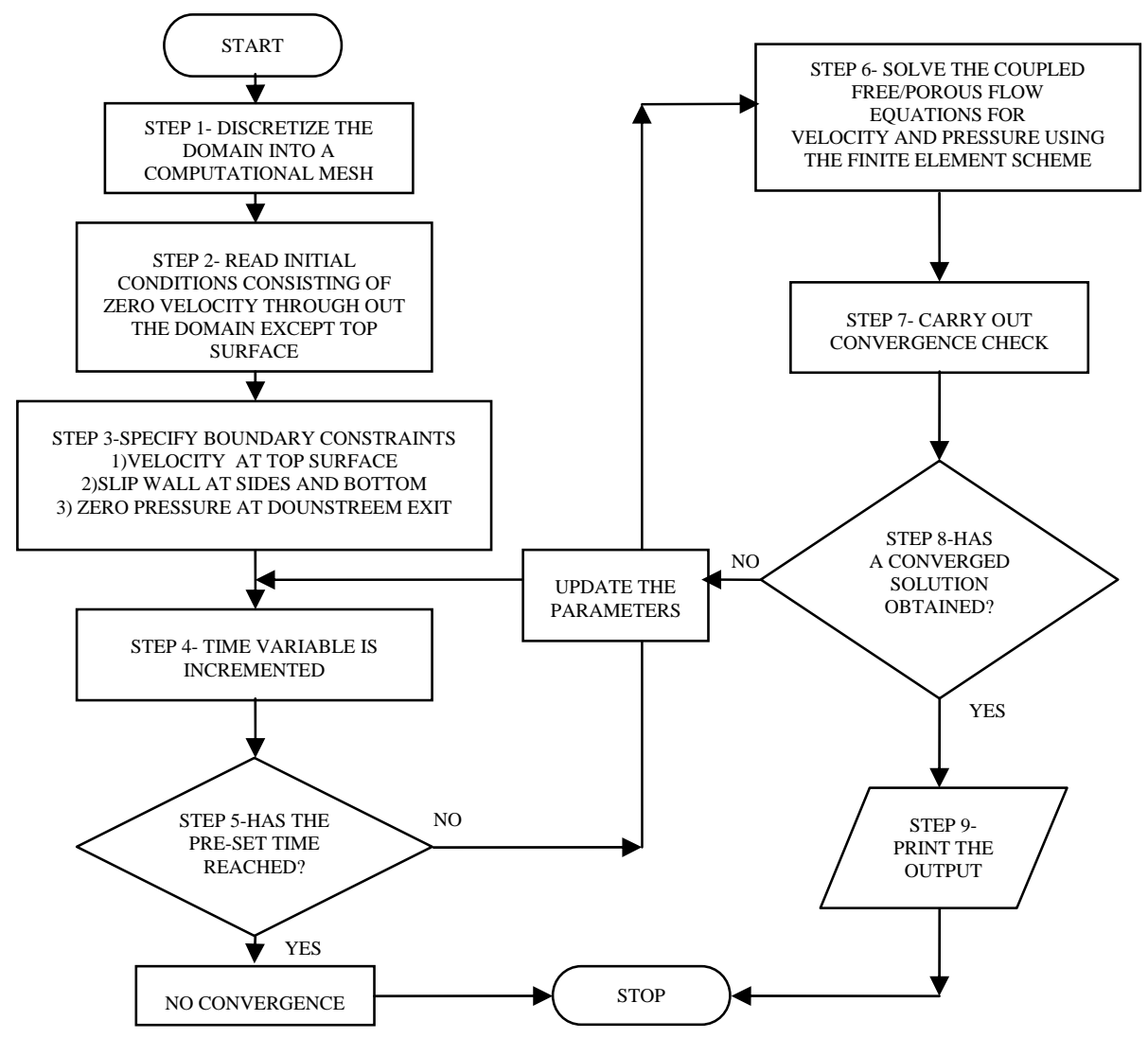

Figure 1: The solution algorithm

\section{COMPUTATIONAL RESULTS AND DISCUSSION}

In this section the results obtained for a block domain subject to varying surface slope are presented and discussed.

In these simulations, the fluid under consideration is water with properties at $20^{0} \mathrm{C}$, as viscosity $=$ $0.001 \mathrm{Kgm}^{-1} \mathrm{~s}^{-1}$ and density $=1000 \mathrm{Kgm}^{-3}$. The velocity of sound in water is taken to be approximately as $10000 \mathrm{~ms}^{-1}$. Depending upon the property of the permeable medium (which can be 
isotropic or anisotropic), appropriate values of permeability are used to cover a range of realistic situations. The time stepping parameter $(\alpha)$ in the present scheme is 0.95 and the time increment $(\Delta t)$ is 20 seconds.

\section{Block domain consisting of isotropic permeable medium}

A block domain of $60 \mathrm{~m}$ width $(\mathrm{W})$ x $60 \mathrm{~m}$ height $(\mathrm{H})$ along $\mathrm{x}, \mathrm{y}$ in one side of the domain and 60 $\mathrm{m}(\mathrm{W})$ x $30 \mathrm{~m}(\mathrm{H})$ along $\mathrm{x}, \mathrm{y}$ in the other side of the domain and 200m length $(\mathrm{L})$ along $\mathrm{z}$ axis is modelled. Therefore initially a surface slope of $8.53^{\circ}$ is considered. The magnitude of surface velocity is $0.2 \mathrm{~ms}^{-1}$. Initially a computational grid of 5760 eight noded brick elements and 6929 nodes shown in figure 2 . This grid is obtained by the division of the domain in $40 \times 12 \times 12$ element in $\mathrm{x}, \mathrm{y}, \mathrm{z}$ direction respectively. The permeability coefficient for this homogeneous isotropic porous domain is taken as: $\left(K_{x x}=K_{y y}=K_{z z}=10^{-9} \mathrm{~m}^{2}\right)$

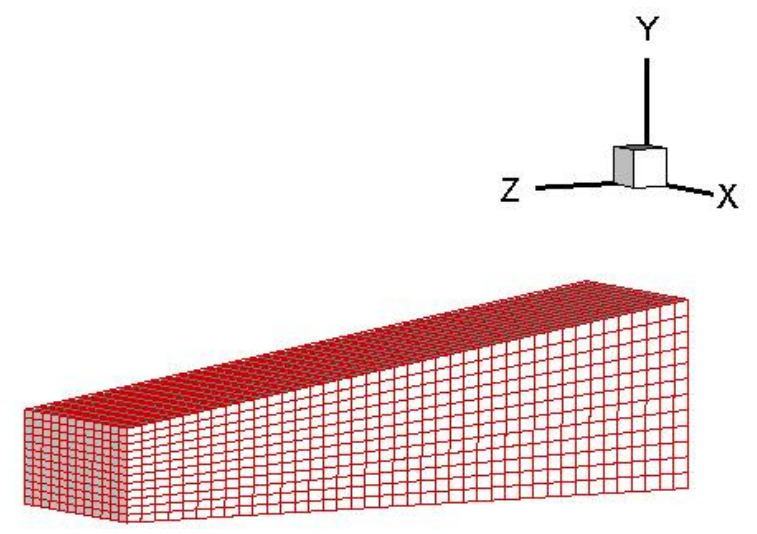

Figure 2: Finite element mesh (12x12x40 division)

The schematic representations of the boundary conditions imposed over the different sides of this domain are shown in figures 3 and 4.

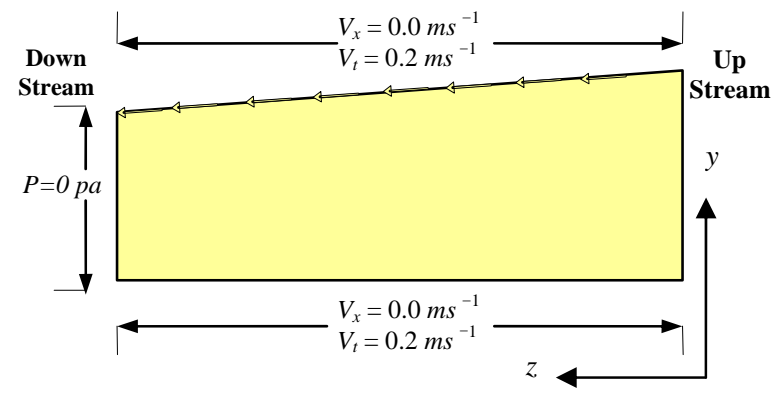

Figure 3: Schematic representation of the boundary condition in the $y \mathrm{z}$ plane

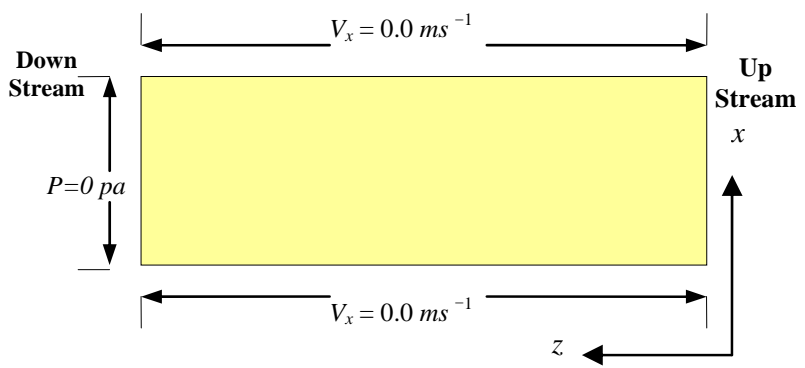

Figure 4: Schematic representation of the boundary condition in the $x \mathrm{z}$ plane 
In addition to the top surface flow velocity $\left(0.2 \mathrm{~ms}^{-1}\right)$ at the downstream exit a zero pressure datum is prescribed. Slip wall boundary conditions are imposed on the sides of the domain. This means that velocity components vertical to each wall is set to be zero whilst the other components are left to be free.

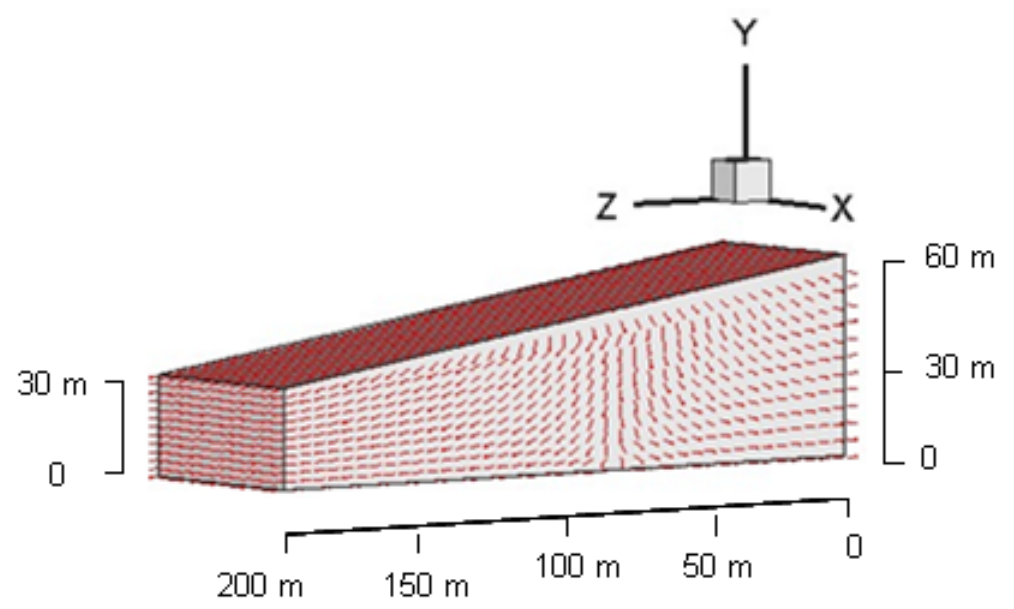

Figure 5: Simulated velocity field visible on the side wall and exit surfaces(surface slope $8.53^{\circ}$ )

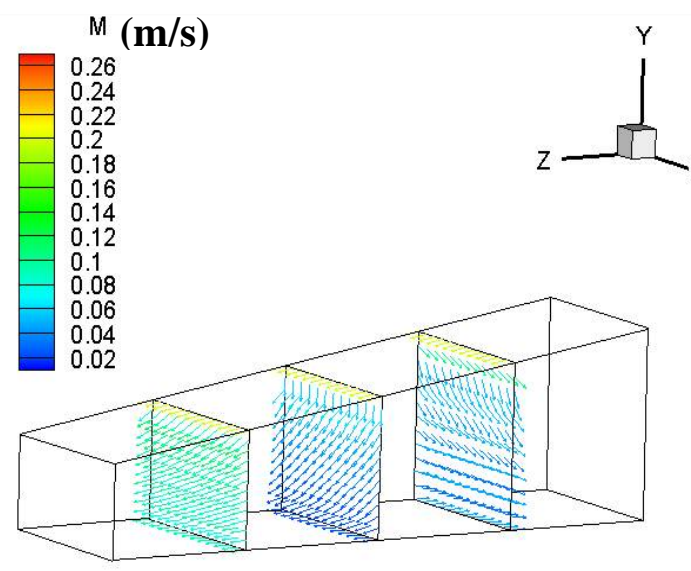

Figure 6: Simulated velocity field on three sample cross sections(surface slope $8.53^{\circ}$ ) $\mathrm{M}$ on colour charts represents magnitude of velocity

To make the representation of the velocity field clearer, only the computed velocity vectors on three sample cross sections are shown in figure 6. The corresponding nodal pressures and magnitude of velocity are shown in figures 7 and 8, respectively. This result shows that, as intended, viscous stress associated with the bulk matrix of the fluid is transferred to the solid porous matrix.

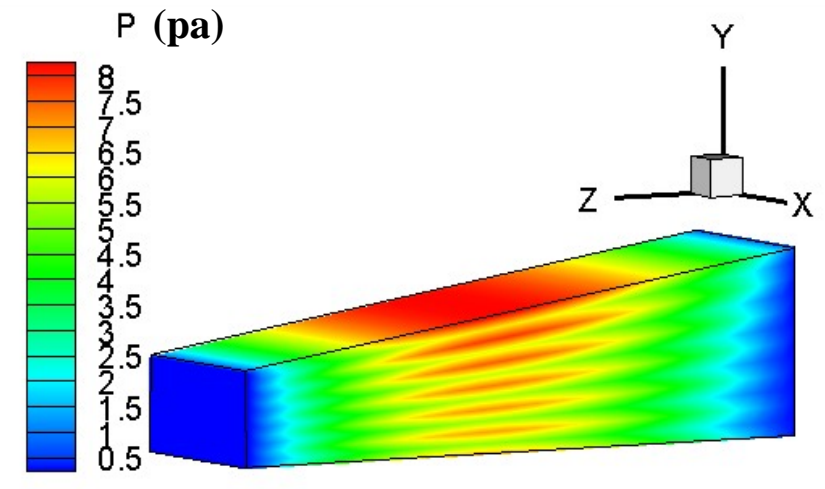

Figure 7: Simulated pressure field(surface slope $8.53^{\circ}$ ) 
The simulated pressure field shown in figure 7 corresponds to a layered flow regime. This shows that the fluid flowing over surface of a saturated layer results in a disturbance in the hydraulic equilibrium of the domain immediately under that surface. Fluid flow in this domain in turn affects the layer under it. This process is repeated layer by layer within the domain. The simulated pressure field closely corresponds with the velocity field pattern shown in figure 5 . The close correspondence between the pressure and velocity fields provides an indication for the self consistency of the simulated results.

To demonstrate the velocity filed pattern within the domain we consider a cross section as shown in figure 8. This figure again confirms that despite the ground being saturated significant flow under the ground is generated by the imposed surface flow which can potentially lead to the transport of pollutants under the ground due to surface run off flow.

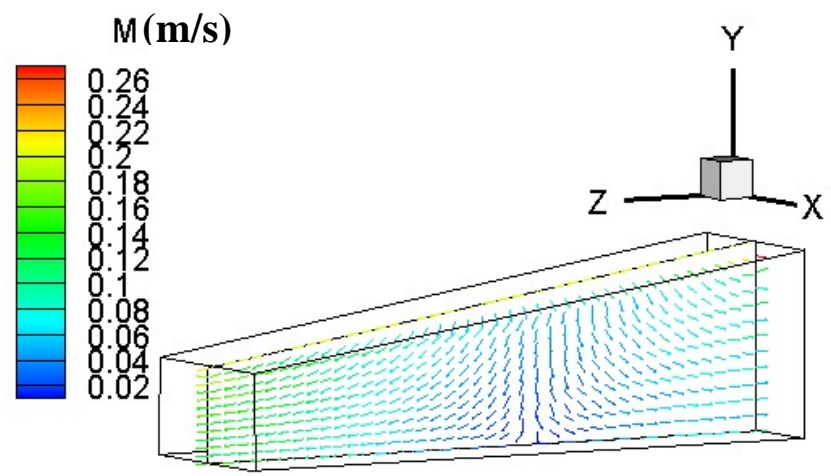

Figure 8: Velocity vector in the section parallel to the $z \mathrm{y}$ plane at $\mathrm{x}=30 \mathrm{~m}$ in the domain $\mathrm{M}$ on colour charts represents magnitude of velocity(surface slope $8.53^{\circ}$ )

To study the influence of the surface slope on the intensity of underground circulation caused by the surface flow, a second domain which has a different surface slope of $12.68^{\circ}$ is considered. All other boundary conditions are kept to be similar to the previous case. Simulation results shown in figures 10 and 11 clearly indicate the strong relation between the surface slope and the underground circulations caused by the surface flow. For example despite a pattern of circulation similar to the previous case, velocities have increased by as much as $38 \%$.

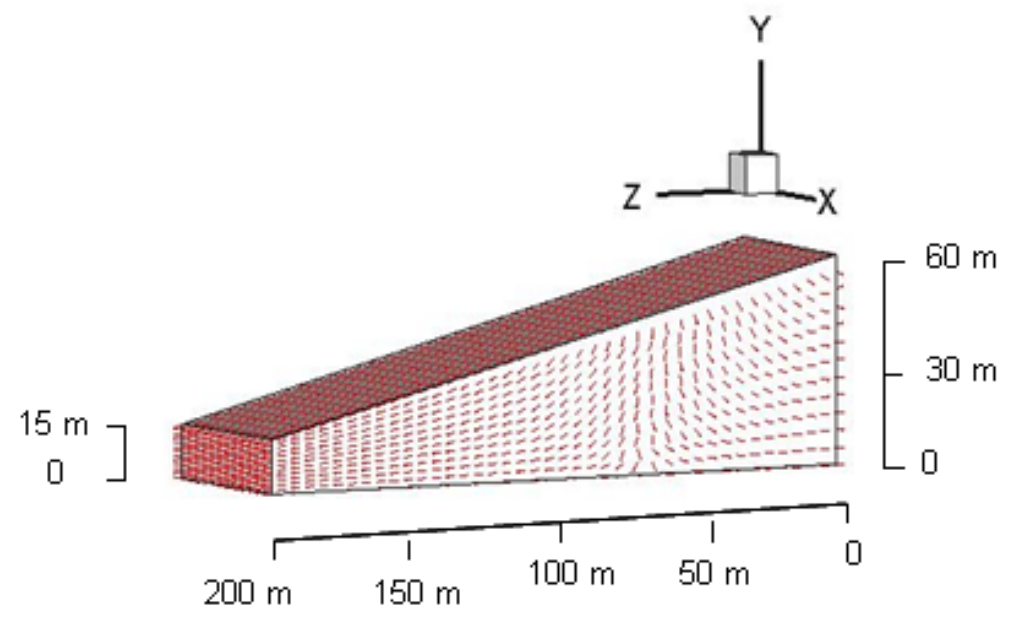

Figure 9: Simulated velocity field visible on the side wall and exit surfaces (surface slope $12.68^{\circ}$ ) 


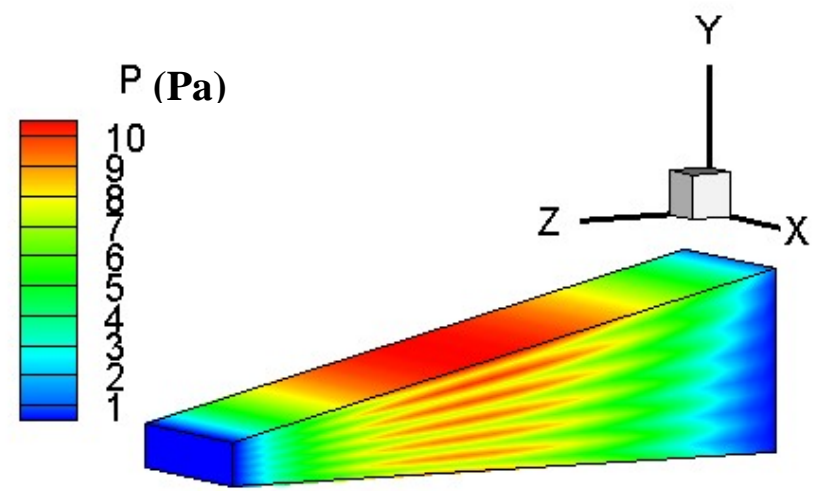

Figure 10: Simulated pressure field(surface slope $12.68^{\circ}$ )

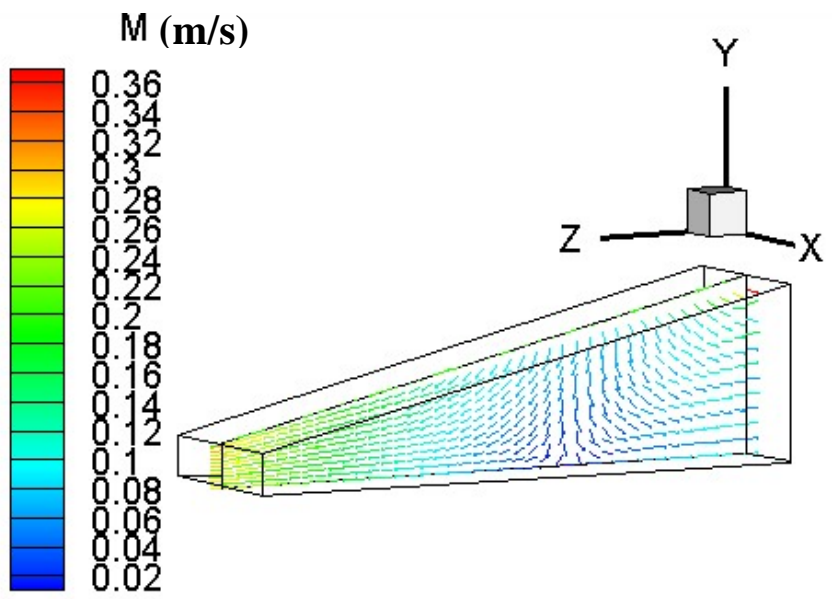

Figure 11: Simulated flow pattern over a cross section parallel to the $\mathrm{z} y$ plane at $\mathrm{x}=30 \mathrm{~m}$ $\mathrm{M}$ on colour charts represents magnitude of velocity(surface slope $12.68^{\circ}$ )

The simulations do not show any sign of instability or any other numerical problems. In order to confirm the convergence of the results shown in this paper all of the described simulations were repeated using a different mesh configuration obtained by 12x12x50 division of the block domain along $\mathrm{x}, \mathrm{y}$ and $\mathrm{z}$ axis, respectively. Simulation results obtained using this mesh were identical to the previous case shown in this paper proving the independence of the results from the computational grid.

\section{CONCLUSIONS}

In this work we have shown that flow over the surface of a saturated land results in the establishment of a strong layered flow regime under the ground. The mechanism causing this pattern of flow can be explained in terms of the disturbance on the hydraulic equilibrium under a flowing fluid layer. The finite element scheme presented in this work provides means of quantitative analysis of this type of flow and showing that underground flow generated in this manner can be in all directions of decreasing pressure head. Environmental impact of flow over saturated lands often caused by rainfall can therefore be, potentially, very significant because any pollutant under the ground can spread in all directions. As shown in this work factors such as surface slope and obviously land permeability or intensity of surface flow all influence the outcome of such a situation. There is no theoretical difficulty which can be envisaged to preclude the extension of this work to heterogeneous soils as the developed finite element scheme can easily cope with variable permeability. However, before starting such a simulation extensive field survey data are required to obtain meaningful predictions for realistic cases. 


\section{REFERENCES.}

Bear, J. \& Buchlin, J. 1991, Modelling and applications of transport phenomena in porous media, Kluwer Academic Pub.

Bear, J. 1996, "Modeling transport phenomena in porous media", IMA VOLUMES IN MATHEMATICS AND ITS APPLICATIONS, , pp. 27-64.

Bell, F.G. 1992, Engineering properties of soils and rocks, 3rd edn, Butterworth-Heinemann, Oxford ; Boston.

Brinkman, H.C. 1949, "On the permeability of media consisting of closely packed porous particles", Applied Scientific Research, vol. 1, no. 1, pp. 81-86.

Cedergren, H.R. 1989, "Seepage, Drainage, and Flow Nets", John Wiley and Sons, New York New York.

Darcy, H. 1856, "Les fontaines publiques de la ville de Dijon. Exposition et application á suivre et des formules á employer dans les questions de duistribution d'eau", .

Das, D.B. 2002, "Development of a new mathematical model for subsurface water quality management", Water science and technology : a journal of the International Association on Water Pollution Research., vol. 45, pp. 301-308.

Das, D.B. 2001, "LANDFLOW: a 3D finite volume model of combined free and porous flow of water in contaminated land sites", Water science and technology : a journal of the International Association on Water Pollution Research., vol. 43, pp. 55-64.

Das, D.B., Nassehi, V. \& Wakeman, R.J. 2002, "A finite volume model for the hydrodynamics of combined free and porous flow in sub-surface regions", Advances in Environmental Research, vol. 7, no. 1, pp. 35-58.

ISHIZAWA, S. \& HORI, E. 1966, "The Flow of a Viscous Fluid through a Porous Wall into a Narrow Gap: A Consideration of the Slip of Fluid on a Porous Wall Surface", Bulletin of JSME, vol. 9, no. 36, pp. 719-730.

Kulkarni, A., Kaveh-Baghbaderani, B. \& Nassehi, V. 2008, "Finite element modelling of underground water flow in low permeability lands", 6th International Industrial Simulation Conference 2008, , pp. 365-370.

Lage, J. 1998, "The fundamental theory of flow through permeable media from Darcy to turbulence", Transport phenomena in porous media, vol. 2.

Nassehi, V. "Practical Aspects of Finite Element Modelling of Polymer Processing. 2002", .

Nassehi, V. 2007, Computational methods in the management of hydro-environmental systems, IWA, London.

Nield, D.A. 1992, Convection in porous media, Springer-Verlag, New York. 
Reddi, L.N. 2003, Seepage in soils : principles and applications, John Wiley, Hoboken, N.J. ; Great Britain.

Reddy, J. 1984, An Introduction to the Finite Element Method, McGraw-Hill Companies.

Troldborg, M., Binning, P.J., Nielsen, S., Kjeldsen, P. \& Christensen, A.G. 2009, "Unsaturated zone leaching models for assessing risk to groundwater of contaminated sites", Journal of contaminant hydrology, vol. 105, no. 1-2, pp. 28-37.

Uromeihy, A. 2007, "Evaluation and treatment of seepage problems at Chapar-Abad Dam, Iran", Engineering geology., vol. 91, no. 2, pp. 219.

Wakeman, R.J. 2005, Solid /liquid separation principles of industrial filtration, Elsevier, Oxford.

Zienkiewicz, O.C. 2005, The Finite Element Method : Its Basis and Fundamentals, Elsevier, Burlington.

Zienkiewicz, O.C. 2005, The finite element method for fluid dynamics, Butterworth-Heinemann, Oxford.

Zienkiewicz, O.C. 1991, "Incompressibility Without Tears-- How to Avoid Restrictions of Mixed Formulation", International journal for numerical methods in engineering., vol. 32, pp. 1189. 